\title{
On Algorithm for Estimation of Selecting Core
}

\author{
Youngjin $\mathrm{Ahn}^{1}$, Moonseong $\mathrm{Kim}^{1}$, \\ Young-Cheol Bang ${ }^{2}$, and Hyunseung Choo ${ }^{1}$ \\ 1 School of Information and Communication Engineering, \\ Sungkyunkwan University, 440-746, Suwon, Korea +82-31-290-7145 \\ watchman@skku.edu \\ \{moonseong, choo\}@ece.skku.ac.kr \\ 2 Department of Computer Engineering,
}

Korea Polytechnic University, 429-793, Gyeonggi-Do, Korea +82-31-496-8292

ybang@kpu.ac. kr

\begin{abstract}
With the development of the multicast technology, the realtime strategy among the group applications using the multicast routing is getting more important. An essential factor of these real-time applications is to optimize the Delay- and delay Variation-Bounded Multicast Tree (DVBMT) problem. In this paper, we propose Estimation of Selecting Core (ESC) algorithm for DVBMT solution. Furthermore, it is expected that ESC algorithm results in the better any other DVBMT solutions.
\end{abstract}

\section{Introduction}

For the QoS transmission, not only the tree cost as a measure of bandwidth efficiency is one of the important factors, but also networks supporting real-time traffic are required to receive messages from source node in a limited amount of time. Therefore, we should consider the multicast end-to-end delay and delay variation problem [2]. In this paper, we study the delay variation problem under the upper bound on the multicast end-to-end delay. We propose an efficient algorithm in comparison with the Delay and Delay Variation Constraint Algorithm (DDVCA [3]), known as the best algorithm so far. Even if the time complexity of our algorithm is same as the one of DDVCA, the proposed algorithm would have the better performance than DDVCA has in terms of the multicast delay variation. The rest of the paper is organized as follows. In Section 2, we state the network model for the multicast routing, and the problem formulation. Section 3 presents the details of the proposed algorithm. Finally, section 4 concludes this paper.

\section{Network Model for Multicasting and DVBMT}

We consider that a computer network is represented by a directed graph $G=$ $(V, E)$ with $n$ nodes and $l$ links or arcs, where $V$ is a set of nodes and $E$ is a set

\footnotetext{
* This work was supported in parts by Brain Korea 21 and the Ministry of Information and Communication in Republic of Korea. Dr. H. Choo is the corresponding author.
} 
of links, respectively. Each link $e=(i, j) \in E$ is associated with delay $d(e) \geq 0$. The delay of a link, $d(e)$, is the sum of the perceived queueing delay, transmission delay, and propagation delay. We define a path as sequence of links such that $(u, i),(i, j), \ldots,(k, v)$, belongs to $E$.

Let $P(u, v)=\{(u, i),(i, j), \ldots,(k, v)\}$ denote the path from node $u$ to node $v$. If all $u, i, j, \ldots, k, v$ are distinct, then we say that it is a simple directed path. For a given source node $s \in V$ and a destination node $d \in V,\left(2^{s \rightarrow d}, \infty\right)$ is the set of all possible paths from $s$ to $d$.

$$
\left(2^{s \rightarrow d}, \infty\right)=\left\{P_{k}(s, d) \mid \text { all possible paths from } s \text { to } d,{ }^{\forall} s, d \in V,{ }^{\forall} k \in \Lambda\right\}
$$

where $\Lambda$ is an index set. The path-delay of $P_{k}$ is given by $\phi_{D}\left(P_{k}\right)=\sum_{e \in P_{k}} d(e)$, ${ }^{\forall} P_{k} \in\left(2^{s \rightarrow d}, \infty\right) .\left(2^{s \rightarrow d}, \Delta\right)$ is the set of paths from $s$ to $d$ for which the end-toend delay is bounded by $\Delta$. Therefore $\left(2^{s \rightarrow d}, \Delta\right) \subseteq\left(2^{s \rightarrow d}, \infty\right)$.

For the multicast communications, messages need to be delivered to all receivers in the set $M \subseteq V \backslash\{s\}$ which is called the multicast group, where $|M|=m$. The path traversed by messages from the source $s$ to a multicast receiver, $m_{i}$, is given by $P\left(s, m_{i}\right)$. Thus multicast routing tree can be defined as $T(s, M)=\bigcup_{m_{i} \in M} P\left(s, m_{i}\right)$ and the messages are sent from $s$ to $M$ through $T(s, M)$.

The multicast end-to-end delay constraint, $\Delta$, represents an upper bound on the acceptable end-to-end delay along any path from source node to a destination node. The multicast delay variation, $\delta$, is the maximum difference between the end-to-end delays along the paths from the source to any two destination nodes.

$$
\delta=\max \left\{\left|\phi_{D}\left(P\left(s, m_{i}\right)\right)-\phi_{D}\left(P\left(s, m_{j}\right)\right)\right|,{ }^{\forall} m_{i}, m_{j} \in M, i \neq j\right\}
$$

The issue defined and discussed in [2], initially, is to minimize multicast delay variation under multicast end-to-end delay constraint. The authors referred to this problem as Delay- and delay Variation-Bounded Multicast Tree (DVBMT) problem. The DVBMT problem is to find the tree that satisfies

$$
\min \left\{\delta_{\alpha} \mid{ }^{\forall} P\left(s, m_{i}\right) \in\left(2^{s \rightarrow m_{i}}, \Delta\right),{ }^{\forall} P\left(s, m_{i}\right) \subseteq T_{\alpha},{ }^{\forall} m_{i} \in M^{\forall} \alpha \in \Lambda\right\},
$$

where $T_{\alpha}$ denotes any multicast tree spanning $M \cup\{s\}$.

\section{The Proposed ESC Algorithm}

Description of ESC Algorithm: We designate the proposed algorithm as Estimation of Selecting Core (ESC). ESC algorithm gives an explicit solution about the DVBMT problem. We define the MODE function, since the location of the core node influences the multicast delay variation. In addition to the $M O D E$ function, we measure the delay variation for each mode, using the $C M P$ function. The $M O D E$ and $C M P$ functions are deployed as follows. 


$$
M O D E(c)=\left\{\begin{array}{l}
\text { I } \quad \text { if } c=s \\
\text { II } \text { if }{ }^{\exists} m \text { in } P(s, c), \text { where }{ }^{\forall} m \in M \\
\text { III if }{ }^{\exists} s \text { in } P(m, c), \text { where }{ }^{\forall} m \in M \\
\text { IV if II and III } \\
\text { V } \quad \text { otherwise }
\end{array}\right.
$$

where $s$ is source node.

$$
C M P(x)=\left\{\begin{array}{ll}
\mid\left(d s_{\text {core }}+\text { max_delay }\right)-d s_{m_{k}} \mid & \text { if } x=\text { II or III or IV } \\
d v_{\text {core }} & \text { otherwise }
\end{array},\right.
$$

where core $=M O D E^{-1}(x), \max \_$delay $=\max \left\{\min \left\{\phi_{D}(P(\right.\right.$ core,$\left.m))\right\} \mid$ ${ }^{\forall} m \in M \backslash\left\{m^{*} \in M{ }^{\exists} m^{*}\right.$ in $P(s$, core $) \bigvee{ }^{\exists} s$ in $P\left(m^{*}\right.$, core $\left.\left.)\right\}\right\}$. $d s_{v_{i}}$ and $d v_{v_{i}}$ are defined in Fig. 1.

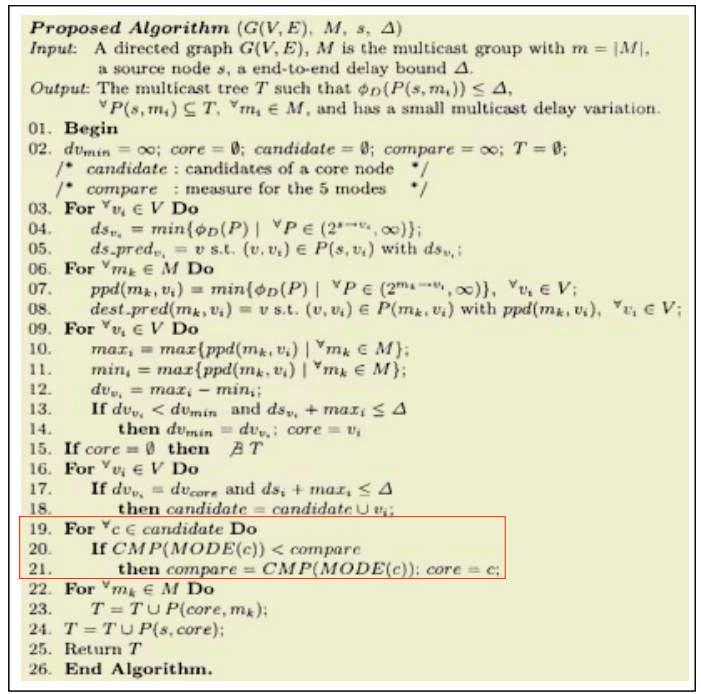

Fig. 1. Pseudo Code of ESC Algorithm

In Fig. 1, Lines 9-21 gives an account of the process to search the suitable core node. Giving a specification, Line 9-15 picks out the minimum $d v_{v_{i}}$, comparing the difference between the maximum delay variation and the minimum delay variation for each node, and verifying the upper bound $\Delta$. If there isn't such a core node, the algorithm will be terminated because the multicast tree cannot be constructed within $\Delta$. In Lines 16-18, ESC algorithm stores the candidate cores, after looking for the node which has the same value as minimum $d v_{v_{i}}$. Lines 19-21 finds out the best core that has the minimum delay variation, using the measure $C M P$ for the 5 modes. These cases are described as follows. The total time complexity of the proposed algorithm is $O\left(m n^{2}\right)$, being equal to DDVCA. 
Five-Mode Core Selection: As above functions $M O D E$ and $C M P, M O D E$ I is that the core node corresponds to the source node. In this case, $C M P$ keeps $d v_{\text {core. }}$ Otherwise, $M O D E \mathrm{~s} \mathrm{II} \sim \mathrm{V}$ are executed.

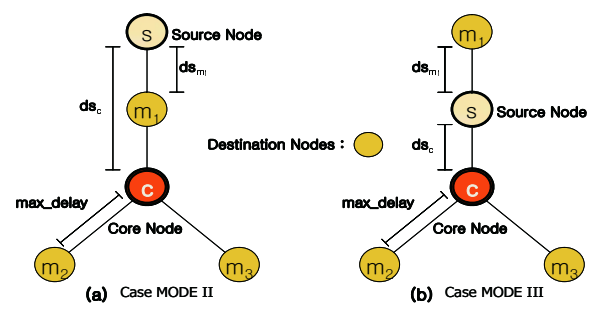

Fig. 2. Main Idea of Mode Function

In the case of $M O D E$ II [1], Fig. 2 (a) shows that there exists the destination node from the source node to the core node. $C M P$ which can be computed stores the sum of the delay from the core node to the destination node and max_delay. The third case just satisfies MODE III. In this case, ESC algorithm initially adds the delay from the source node to the core node to max_delay and subtracts the delay from the source node to the adjacent destination node, and stores the value with $C M P$. If the value is negative, $C M P$ accepts the absolute value of it. The fourth case, MODE which satisfies II and III should find the factor to determine $C M P$, having situation that the destination nodes exist around the source node. The measure of the comparison is determined with the minimum delay from the source to the associated destinations. We can decide this situation because the shortest delay value from the source node affects the delay variation of the created tree. Therefore, ESC algorithm selects either MODE II or III. In $M O D E \mathrm{~V}$, if ESC algorithm doesn't satisfy MODEs I IV, CMP stores $d v_{\text {core. }}$

\section{Conclusion}

In this paper, we consider the transmission of a message that guarantees certain bounds of the end-to-end delays as well as the multicast delay variations computer network. The time complexity of ESC algorithm is $O\left(m n^{2}\right)$, which is the same as that of DDVCA. Furthermore, it is expected that ESC algorithm results in the better minimum multicast delay variation than DDVCA.

\section{References}

1. M. Kim, Y.-C. Bang, and H. Choo, "Efficient Algorithm for Reducing Delay Variation on Bounded Multicast Trees," Springer-Verlag Lecture Notes in Computer Science, vol. 3090, pp. 440-450, September 2004. 
2. G. N. Rouskas and I. Baldine, "Multicast routing with end-to-end delay and delay variation constraints," IEEE JSAC, vol. 15, no. 3, pp. 346-356, April 1997.

3. P.-R. Sheu and S.-T. Chen, "A fast and efficient heuristic algorithm for the delayand delay variation bound multicast tree problem," Information Networking, Proc. ICOIN-15, pp. 611-618, January 2001. 